\title{
The Personalized Shoulder Reconstruction Assisted by 3D Printing Technology After Resection of the Proximal Humerus Tumours
}

This article was published in the following Dove Press journal: Cancer Management and Research

\author{
Hongzhi Hu',* \\ Weijian Liu (D) ${ }^{1, *}$ \\ Qianwen Zeng ${ }^{2, *}$ \\ Shangyu Wang' \\ Zhicai Zhang' \\ Jianxiang Liu (1)' \\ Yingze Zhang ${ }^{3}$ \\ Zengwu Shao' \\ Baichuan Wang' \\ 'Department of Orthopaedics, Union \\ Hospital, Tongji Medical College, \\ Huazhong University of Science and \\ Technology, Wuhan 430022, People's \\ Republic of China; ${ }^{2}$ Department of \\ Pediatrics, Tongji Hospital of Tongji \\ Medical College of Huazhong University \\ of Science and Technology, Wuhan \\ 430030, People's Republic of China; \\ ${ }^{3}$ Department of Orthopedic Surgery, The \\ Third Hospital of Hebei Medical \\ University, Qiaoxi District, Shijiazhuang, \\ Hebei, 05005I, People's Republic of \\ China, Key Laboratory of Biomechanics \\ of Hebei Province, Shijiazhuang, Hebei \\ 05005I, People's Republic of China
}

*These authors contributed equally to this work

\begin{abstract}
Background: The reverse shoulder arthroplasty (RSA) may be a promising alternative for proximal humerus tumours because of good postoperative shoulder function. However, the conventional reverse shoulder prosthesis can not meet individual needs and RSA has been associated with a relatively high complication rate. Therefore, implant design and surgical reconstruction technique warrant further study.
\end{abstract}

Methods: Between September 2015 and May 2018, 7 patients were treated via RSA after enbloc resection of the proximal humerus tumours. A 3D-printed guiding baseplate was used to assist the implant of the 3D-printed glenoid prosthesis; a personalized humerus prosthesis was used to reconstruct the proximal humerus. The functional outcomes were assessed by range of motion (ROM) of the shoulder joint, Musculoskeletal Tumour Society (MSTS) functional score, and Toronto Extremity Salvage Score (TESS). We also analyzed tumour recurrence, metastases, and complications associated with the reconstruction procedure.

Results: All patients were observed for 14 to 36 months, with an average of 23.6 months. At the final follow-up, the mean MSTS score was $85.7 \%$ (range, 73.3-93.3\%), and the mean TESS score was $90.0 \%$ (range, $84.1-95.9 \%$ ). No instability, infection, scapular notching, loosening or fracture were observed in this series. One patient with GCT suffered from pulmonary metastasis, while one with osteosarcoma died because of pulmonary metastasis. Conclusion: The 3D-printed guiding baseplate facilitated the accurate implantation of the glenoid prosthesis. The RSA based on a 3D-printed glenoid prosthesis and a personalized custom-made humerus prosthesis significantly improved the shoulder function and decreased the complication rate. Further studies of a larger scale with longer follow-up are required to validate this technology.

Keywords: 3D printing technology, tumour, proximal humerus, reverse shoulder arthroplasty, reconstruction

\section{Introduction}

The proximal humerus is the most predilection site of bone tumours in the upper extremity. ${ }^{1}$ Currently, limb salvage treatment is the mainstay of treatment for bone tumours of the proximal humerus. However, the function reconstruction after en bloc resection of the proximal humerus tumours remains a surgical challenge because of large bone defects and surgical loss of functional soft tissues. ${ }^{2,3}$

Numerous methods are available for the reconstruction of long bone defects following en bloc resection of the proximal humerus tumours, including vascularized fibular autograft, ${ }^{4}$ osteoarticular allograft, ${ }^{5,6}$ endoprosthetic reconstruction, ${ }^{7,8}$ and
Correspondence: Zengwu Shao; Baichuan Wang Hospital, Tongji Medical College,

Huazhong University of Science and

Technology, Wuhan 430022, People's

Republic of China

Tel +86 I397I 021748

Tel +86 I8062763619

Email szwpro@163.com;

wangbaichuan-II2@I63.com 
allograft-prosthetic composite arthroplasty. ${ }^{9-11}$ Nevertheless, these techniques are usually associated with bone absorption, nonunion, shoulder joint dislocation and poor shoulder function. More recently, more surgeons choose RSA for the shoulder joint reconstruction after resection of the proximal humerus tumours; the reason for this is that it can improve the shoulder function after reconstruction and decrease the complication rate. ${ }^{12,13}$ Initially, RSA, first proposed by Grammont et al, ${ }^{14}$ was devised to treat elderly patients with rotator cuff deficiency in case of failed conservative treatment. $^{15,16}$ After that, the RSA has been used for the treatment of comminuted fracture of the humeral head as well as the proximal humerus tumour. ${ }^{17,18}$ However, the conventional reverse prostheses might be inappropriate for the reconstruction of the long bone defects following en bloc resection of the proximal humerus tumours because of the original design intent. ${ }^{12,13,19}$ Moreover, the reverse prosthesis reconstruction has been associated with a relatively high complication rate. ${ }^{10,18,19}$

The computer-designed and 3D printing technology may provide an alternative. Recently, tumour models, guiding plates and/or prostheses based on 3D printing technology have been used for precise tumour resection and functional reconstruction. $^{20,21}$ In this study, a personalized guiding baseplate was designed to assist the implant of a 3D-printed glenoid prosthesis; a reverse shoulder prosthesis consisting of a 3D-printed glenoid prosthesis and a personalized custom-made humerus prosthesis were used for shoulder joint reconstruction. We elaborated the reconstruction procedure and evaluated functional results, oncological status and the reconstruction-associated complications.

\section{Materials and Methods Clinical Data}

This retrospective study was conducted with the approval of the Ethics committee of Tongji Medical College, Huazhong University of Science and Technology. All the patients gave written informed consent and agreed to publish their images in our study.

From September 2015 to May 2018, seven patients with the proximal humerus tumours underwent en bloc resection of tumours and reconstruction with personalized reverse shoulder prostheses. Of them, three were male and four were female. The average age of the patients at surgery was 34.9 years (range, 16-56 years). The pathological diagnoses were as follows: three osteosarcomas, two chondrosarcomas, and two Campanacci grade III giant cell tumours (GCTs) of bone. All patients underwent preoperative X-ray, thin-slice $(1 \mathrm{~mm})$ computed tomography (CT) scan of shoulder joint and lung, magnetic resonance imaging (MRI) of the involved shoulder joint, and electrical capacitance tomography (ECT) bone scan.

Accurate diagnosis of the tumours depended on a comprehensive analysis of clinical manifestations, imaging features and pathology. The pathology diagnosis was acquired by percutaneous bone needle biopsy. The biopsy track was carefully planned according to the subsequent surgical approach. The three patients with osteosarcoma received neoadjuvant chemotherapy and the other four patients underwent surgical treatment alone.

\section{Prosthesis Design and Fabrication}

The reverse shoulder prosthesis, consisting of a 3D-printed glenoid prosthesis and a custom-made humerus prosthesis, was designed by our professional bone tumour team.

The collected preoperative CT and MRI data were imported into E-3D x64 v13.20 (E-Feature Info. Tech. Co., Ltd, Shanghai, China) in DICOM format to build a 3D computer model in which the tumour extent and osteotomy position were determined (Figure 1A-C). The personalized glenoid prosthesis and humerus prosthesis were then designed, as shown in Figure 1D. The procedures of tumour resection and prosthesis implant were simulated using the E-3D x64 v13.20 (Figure 2A-E). Lastly, the glenoid prosthesis and the guiding baseplate were produced using 3D printing technology (EOS M290, GmbH Electro Optical Systems, Germany; Arcam A1, EBM, Arcam Sweden) (Figure 3A and B), and the humerus prostheses were custom-made conventionally (THYTEC, Co., Ltd, Shanghai, China). The guide plate is made of nylon powder, and the whole prosthesis is made entirely of titanium alloy. The 3D-printed glenoid prosthesis has a pore size of $450-550$ my and a porosity of $60 \%$.

\section{Surgical Procedure}

All the surgeries were performed by the experienced musculoskeletal oncologists (Z. W. S. and B. C. W). After general anaesthesia, all patients were placed in the beach chair position. All operations were performed via a deltopectoral approach. The excision of soft tissue depended on the involvement of the tumours. The axillary nerve was identified and protected carefully during the operation. A jigsaw was used to perform the osteotomy according to the preoperative plan. The previous biopsy track along with the tumour was removed. 

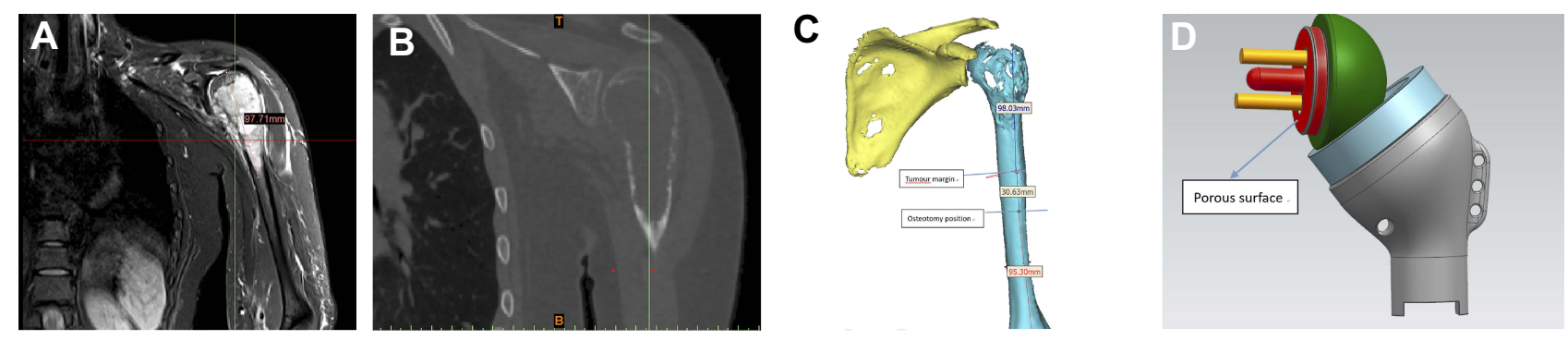

Figure I (A) A coronal contrast-enhanced T2-weighted MRI in a patient with Campanacci grade III GCT of bone; (B) a coronal CT scan images; (C) Based on the preoperative CT and MRI data, the tumour extent and osteotomy position were outlined on a 3D computer model; (D) the design of the 3D-printed glenoid prosthesis and a personalized custom-made humerus prosthesis.

Abbreviation: 3D, three-dimensional.
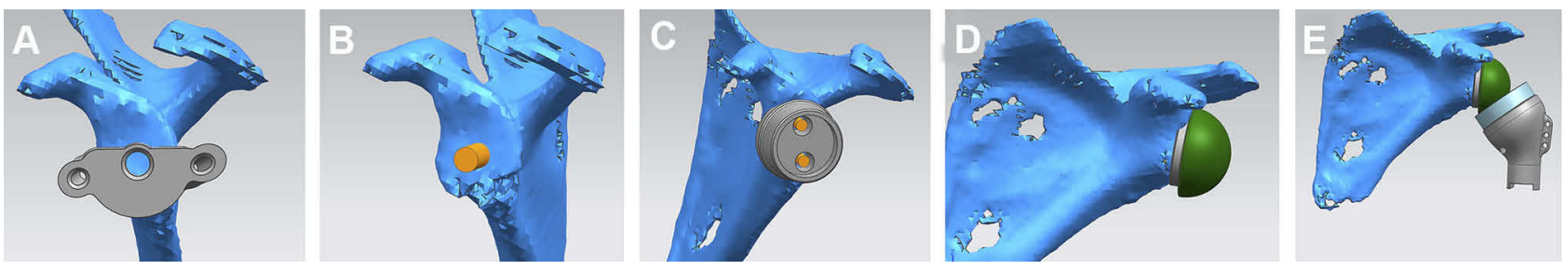

Figure 2 (A-E) The installation and fixation of glenoid implants were simulated on a 3D computer model. Abbreviation: 3D, three-dimensional.

After that, the glenoid was exposed and the 3D-printed guiding baseplate was placed according to the operative plan (the middle and lower third of the glenoid) and was fixed with two Kirschner wires. The guiding baseplate was removed after the position of the glenoid prosthesis being determined, and then a guide pin was inserted and the articular cartilage was removed. After confirming the position, the 3D-printed glenoid prosthesis and the humerus prosthesis were implanted (Figure 3C-E). Lastly, the stability and ROM of the shoulder joint were examined. The remaining rotator cuff was sewed up to the reserved holes of the prosthesis to improve the stability of the shoulder joint.

To decrease the infection rate, the wound was flushed using a pulsing squirt gun during the operation; the prophylactic antibiotics were used $1 \mathrm{hr}$ before surgery and 24 hrs after surgery.

\section{Postoperative Management}

The shoulder joint was maintained on an abduction splint for at least 6 weeks postoperatively. All patients were allowed to perform active movements of the wrist and the elbow and passive exercises of the shoulder on the first day after surgery. After 6 weeks, patients were encouraged to perform active exercises of the shoulder joint to achieve more flexible ROM. The intensity of exercise gradually increased under the supervision of our physio team. The three patients with osteosarcoma received postoperative chemotherapy.

All patients were followed up with clinical and imaging assessments. X-ray of the reconstructed shoulder joint was performed every month for the first 3 months after surgery and then every 3 months thereafter. Chest CT scan was performed every 3 months and bone scan every 6 months to identify potential metastases. At the last followup, active ROM, including abduction, forward flexion, external rotation and internal rotation, was measured and recorded. The MSTS functional score ${ }^{22}$ and TESS score ${ }^{23}$ were also assessed over the course of follow-up.

\section{Results}

All patients were followed up for 14 to 36 months, with an average of 23.6 months. Five patients were alive without any evidence of disease. One patient (Case 4) with GCT of bone was alive but developed pulmonary metastasis (16 months after surgery). One patient (Case 3 ) died due to pulmonary metastasis 15 months after surgery. No local recurrence was observed in our series (Table 1).

All patients underwent en bloc resection of tumours, and the average osteotomy length was $118.6 \mathrm{~cm}$ (range, 106.5-128.7 cm). After surgery, most patients had obvious pain relief. Although one patient complained of mild pain at the final follow-up, the pain can be relieved by nonsteroidal 

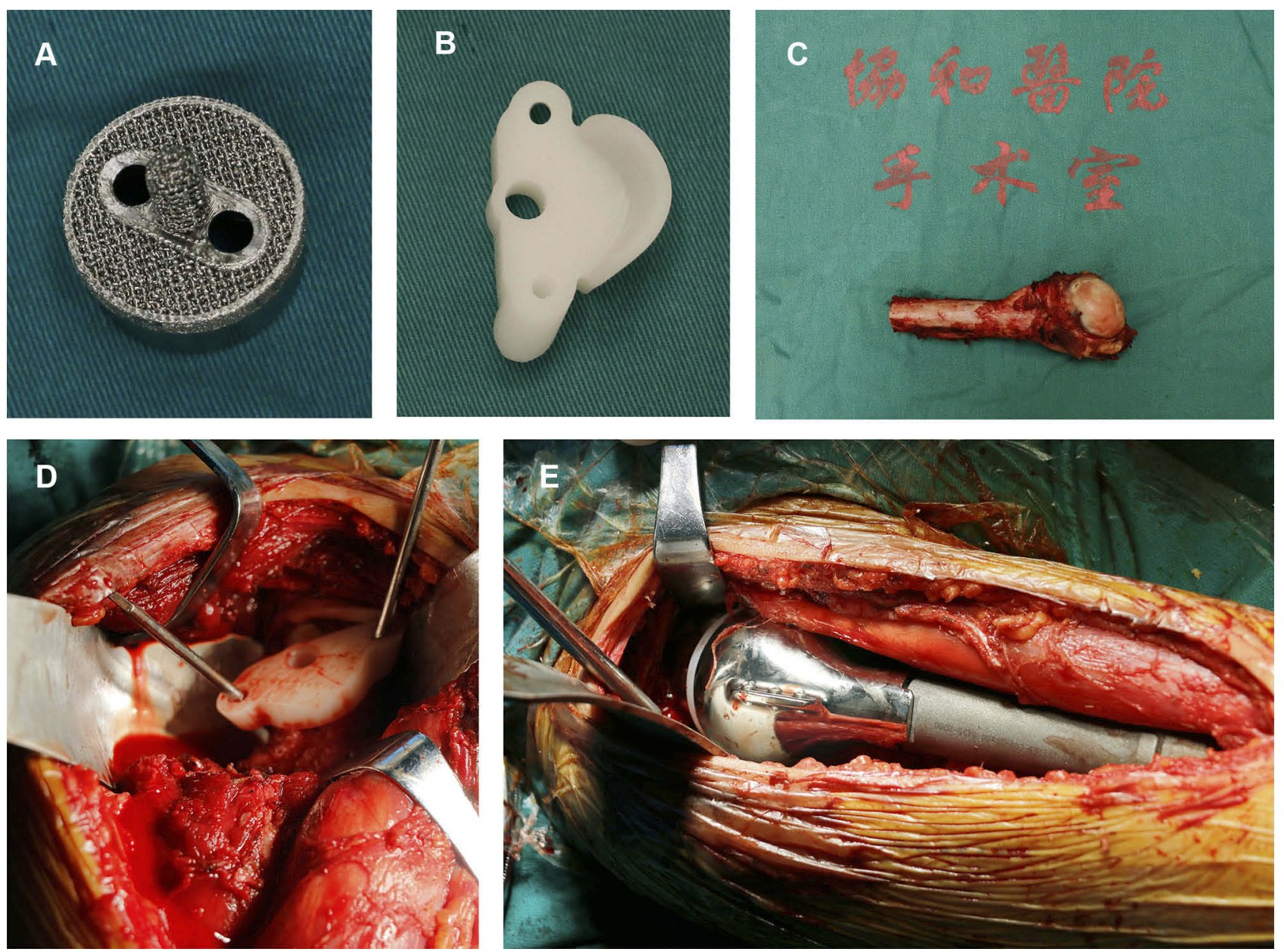

Figure 3 (A) A personalized 3D-printed glenoid prosthesis; (B) a 3D-printed guiding baseplate; (C) en bloc resection specimen of the tumour; (D) the 3D-printed guiding baseplate was placed in the appropriate location (the middle and lower third of the glenoid) and fixed with two Kirschner wires; (E) the custom-made humerus prosthesis was implanted and fixed with cement.

Abbreviation: 3D, three-dimensional.

anti-inflammatory drugs. The functional outcomes allowed patients to return to their normal work and life. At the final follow-up, three patients returned to their previous occupation (light manual worker), and the other three patients had to change their jobs because of the limited ROM of the shoulder joint. The mean MSTS functional outcome score was $85.7 \%$ (range, $73.3 \%$ to $93.3 \%$ ) and the mean TESS score was $90.0 \%$ (range, $84.1 \%$ to $95.9 \%$ ). The mean range of active movement at the final follow-up was forward flexion, $133.4^{\circ}$ (range, $125^{\circ}$ to $150^{\circ}$ ), abduction, $137.6^{\circ}$

Table I Basic Characteristics of All Patients

\begin{tabular}{|l|l|l|l|l|l|l|}
\hline Case & $\begin{array}{l}\text { Gender/Age } \\
\text { (Years) }\end{array}$ & Side & $\begin{array}{l}\text { Pathological } \\
\text { Diagnoses }\end{array}$ & $\begin{array}{l}\text { Follow-Up Time } \\
\text { (Months) }\end{array}$ & $\begin{array}{l}\text { Survival } \\
\text { Status }\end{array}$ & Complication \\
\hline I & M/48 & Left & Chondrosarcoma & 36 & DFS & - \\
2 & F/24 & Right & OS & 32 & DFS & - \\
3 & M/56 & Right & OS & 15 & DOD & Pulmonary metastasis \\
4 & F/23 & Left & GCT & 29 & AWD & Pulmonary metastasis \\
5 & M/32 & Left & GCT & 21 & DFS & - \\
6 & F/45 & Left & Chondrosarcoma & 18 & DFS & - \\
7 & F/l6 & Right & OS & 14 & DFS & - \\
\hline
\end{tabular}

Abbreviations: OS, osteosarcoma; GCT, giant cell tumour; DFS, disease-free survival; DOD, died of disease; AWD, alive with disease. 
(range, $128^{\circ}$ to $150^{\circ}$ ), internal rotation $65.9^{\circ}$ (range, $46^{\circ}$ to $82^{\circ}$ ), and external rotation, $41.9^{\circ}$ (range, $23^{\circ}$ to $65^{\circ}$ ).

During the short-term follow-up period, no complications associated with the reconstruction procedure, such as infection, scapular notching, aseptic loosening, periprosthetic fractures or dislocation, were observed. Two typical cases were shown in Figures 4 and 5.

\section{Discussion}

The proximal humerus is a common site of primary malignant and invasive bone tumours in the upper extremity. The primary aims of the proximal humerus tumours are to remove the tumours completely and preserve the function of the shoulder joint. The functional outcomes of the reconstructed shoulder joint are related to several factors, including whether the rotator cuff and the shoulder girdle muscles are preserved and whether the axillary nerve is involved. ${ }^{18}$ The rotator cuff is known to play an essential role in stabilizing the shoulder joint. ${ }^{24,25}$ However, relevant parts of rotator cuff occasionally have to be sacrificed in order to obtain a wide margin and local oncological control. ${ }^{26}$ Thus, functional reconstruction of the shoulder joint after en bloc resection of the proximal humerus tumours remains a great surgical challenge.

Numerous attempts have been used to reconstruct the functional rotator cuff after en bloc resection of proximal humerus tumours. ${ }^{3,27-29}$ Marulanda et $\mathrm{al}^{27}$ used a synthetic vascular mesh to facilitate soft tissue attachment, which reduced incidence of postoperative dislocation and shoulder instability. In Gosheger et al's study, ${ }^{28}$ they applied a trevira tube to reconstruct the capsule and improve the soft tissue attachment in proximal humerus replacement. Moreover, our previous study also reported a shoulder joint capsule reconstruction procedure using a polypropylene mesh. ${ }^{3}$ However, the shoulder function did not improve as much as predicted previously. Many studies have shown that the RSA can obtain satisfactory shoulder function in patients with rotator cuff excision or dysfunction. ${ }^{19,30}$ The reverse polarity design

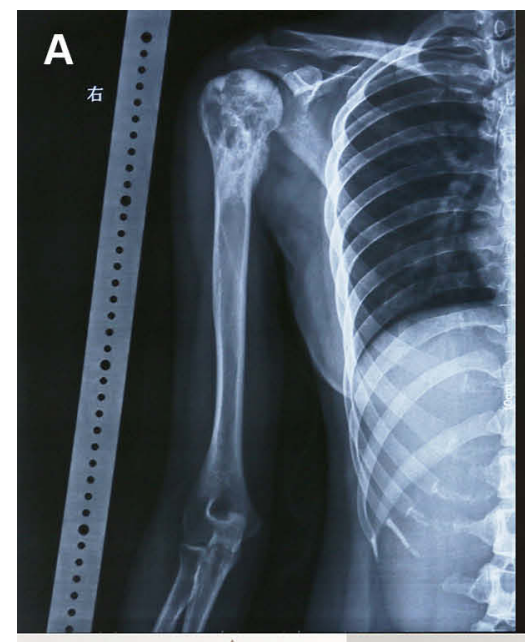

E

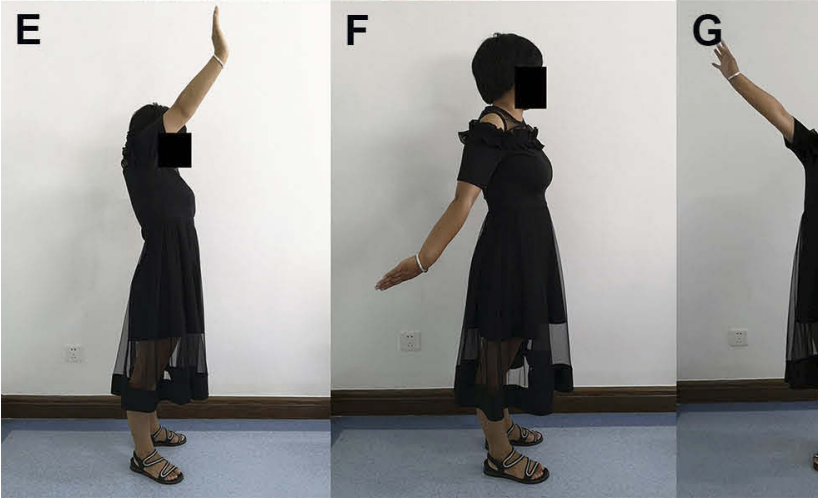

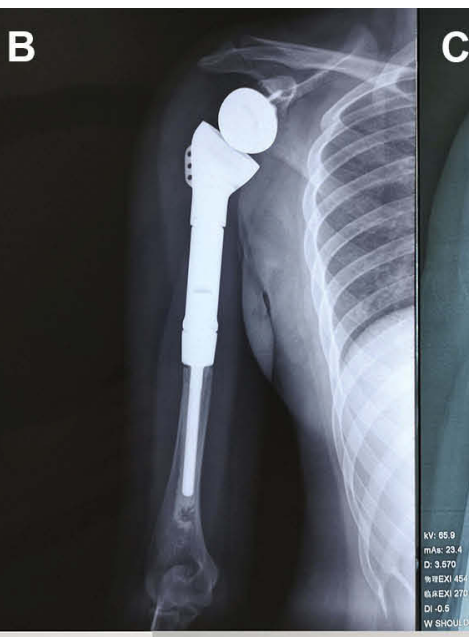

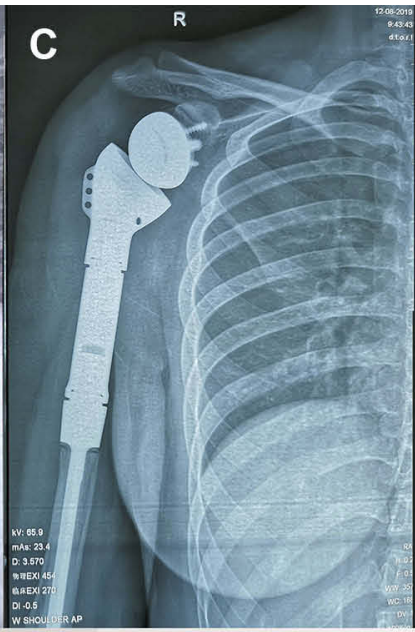

H

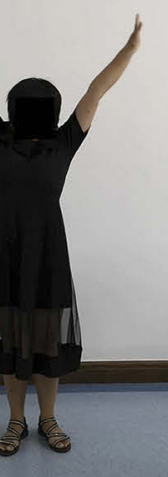

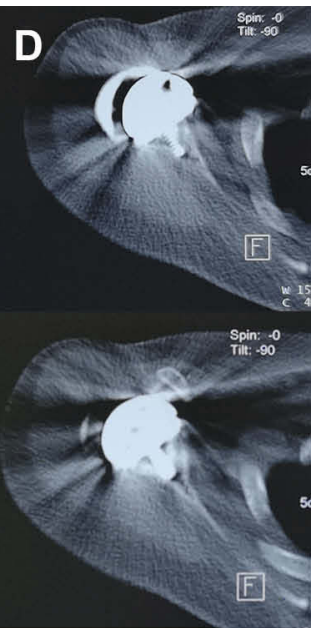

I

Figure 4 A 24-year-old female patient with osteosarcoma underwent RSA following en bloc resection of the right proximal humerus.

Notes: (A) Preoperative X-ray image; (B) postoperative X-ray image 3 months after surgery; (C) postoperative $x$-ray image 16 months after surgery; (D) the transaxial CT scanning of right shoulder joint obtained 10 months after surgery showed osteointegration; (E-I) at the last follow-up, the patient recovered satisfactory contour and function of the shoulder.

Abbreviation: RSA, reverse shoulder arthroplasty. 


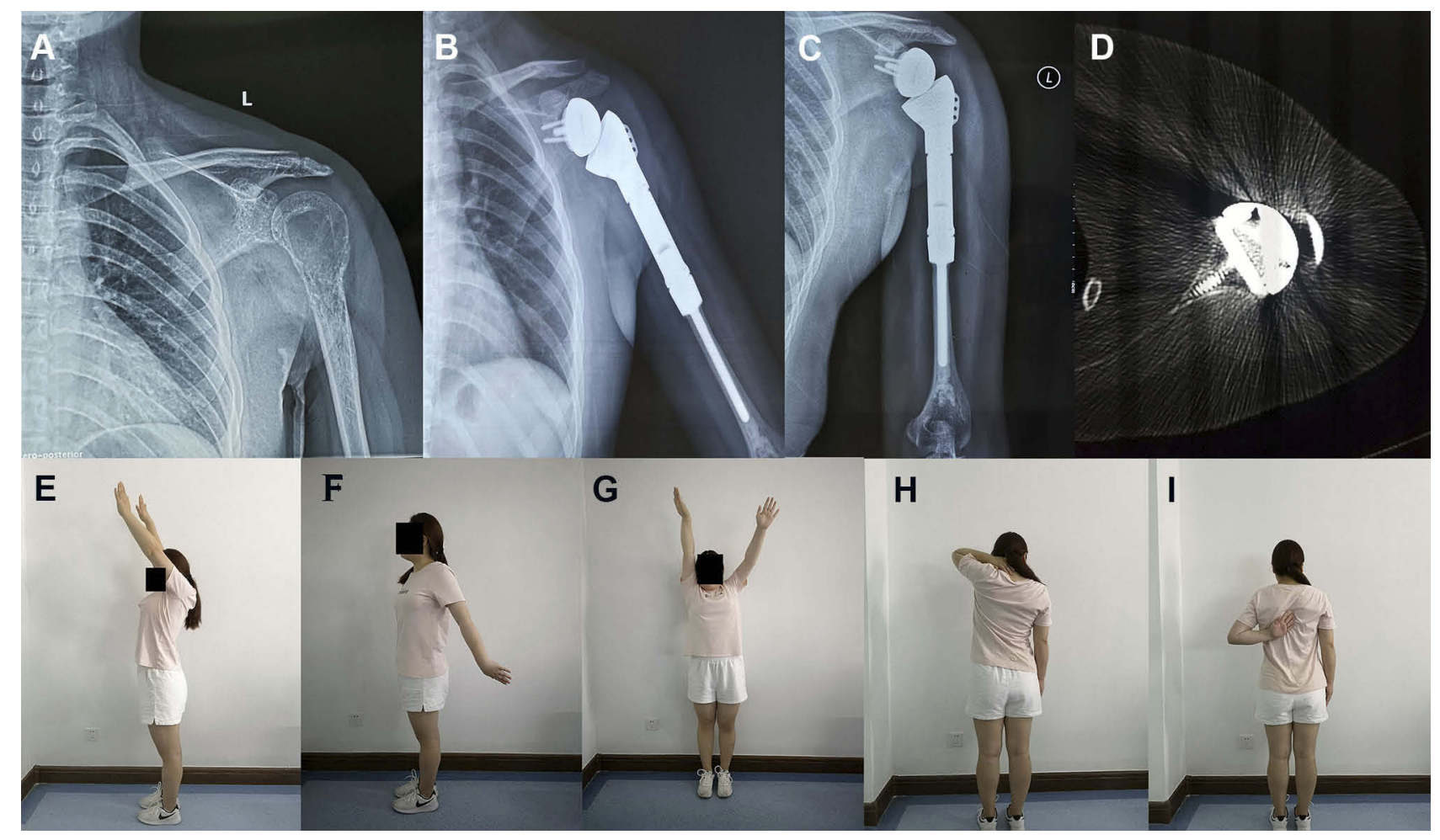

Figure 5 A 23-year-old female patient with Campanacci grade III GCT of bone underwent RSA following en bloc resection of the left proximal humerus.

Notes: (A) Preoperative X-ray image; (B) postoperative X-ray image 5 months after surgery; (C) postoperative X-ray image 12 months after surgery; (D) the transaxial CT scanning of right shoulder joint obtained 16 months after surgery showed osteointegration; (E-I) at the last follow-up, the patient recovered satisfactory contour and function of the shoulder.

Abbreviations: GCT, giant cell tumour; RSA, reverse shoulder arthroplasty.

enables medialization of the centre of rotation and increases the moment arm, which significantly improves the ROM of the shoulder joint. ${ }^{31}$ Recently, RSA has been increasingly used in patients with proximal humeral tumours. ${ }^{12,18,19}$ However, the conventional reverse shoulder prosthesis cannot meet the personalized needs, and RSA has been associated with a relatively high complication rate. ${ }^{10,18,19}$ Reconstruction with the unsuitable prosthesis may result in prosthetic loosening, loss of bone mass and even periprosthetic fractures. ${ }^{25}$ Therefore, appropriate shoulder prosthesis seems to be of particular importance for the postoperative function of patients and long-term survival of prosthesis.

3D printing technology, also referred to additive manufacturing, has gained great attention in the medical field because of its potential benefits. ${ }^{32,33}$ First, 3D-printed realsized models can give the surgeons better visualization and understanding of tumour anatomy. ${ }^{20,34}$ Riggs et $\mathrm{al}^{34}$ reported that they successfully printed a physical 3D-printed model to gain a better understanding of the complex cardiac structures as well as spatial relationship and extent of the tumour before surgery, so as to maximize the removal of the tumour while avoiding damage to critical structures. Second, surgeons can use the 3D-printed surgical guiding plate to assist the osteotomy during the operation, thus improving the accuracy of surgical resection and avoiding larger segmental bone defects. ${ }^{35}$ Also, 3D-printed technology can produce prosthesis with a complex shape precisely matching irregular bone defects, which cannot be easily achieved by traditional manufacturing technology. Moreover, the 3D printing technology allows for the manufacturing of metal prostheses with a porous interfacial layer, which exerts an essential role on the induction of bone ingrowth. ${ }^{36}$

Recently, the model, osteotomy guiding plate and prosthesis based on 3D printing technology have been used for the resection and reconstruction of the bone tumours in various anatomical sites..$^{21,25,37,38}$ In our previous study, with the assistance of 3D-printed pelvic tumour models and osteotomy guiding plates, 3D-printed hemipelvic prostheses were successfully used to reconstruct the defects following the resection of periacetabular malignant bone tumours. ${ }^{21} \mathrm{In}$ this study, seven patients with the proximal humerus tumours were treated by en bloc resection and RAS reconstruction using a 3D-printed glenoid prosthesis and a custom-made humerus prosthesis. A personalized 3D-printed guiding 
baseplate was used for assisting the implant of the glenoid prosthesis. We evaluated functional results, oncological status and the reconstruction-associated complications.

In our series, most patients achieved obvious pain relief at the time of the last follow-up. The functional outcomes allowed the patients to return to their normal life. The mean MSTS functional outcome score was 85.7\% (range, 73.3-93.3\%), and the mean TESS score was $90 \%$ (range, $84.1 \%$ to $95.9 \%$ ). These results were in line with many previous studies in which RSA was employed to treat patients. ${ }^{12,19}$ However, the ROM of the shoulder joint seems to be superior to that of many previous studies. ${ }^{10,19}$ The mean forward flexion, $133.4^{\circ}$ (range, $125^{\circ}$ to $150^{\circ}$ ), abduction, $137.6^{\circ}$ (range, $128^{\circ}$ to $150^{\circ}$ ), internal rotation $65.9^{\circ}$ (range, $46^{\circ}$ to $82^{\circ}$ ), and external rotation, $41.9^{\circ}$ (range, $23^{\circ}$ to $65^{\circ}$ ). The outcomes of ROM may be related to tumour involvement, incision design, different surgeons, reconstruction method and postoperative function exercise.

Scapular notching was one of the most commonly seen complications after RSA. ${ }^{39}$ Bonnevialle et $\mathrm{al}^{19}$ reviewed 10 patients who underwent RSA for the proximal humerus tumours; they found scapular notching in $40 \%$ of the patients during the follow-up. The main cause of scapular notching is that repeated impingement of the humeral prosthesis against the inferior scapular neck during arm abduction, which might be attributed to the relatively centred position of the glenoid component. ${ }^{39-41}$ To minimize the risk of notching, a 3D-printed guiding baseplate was used to determine the optimal position of the glenoid prosthesis. In our patients, no scapular notching was observed. Shoulder instability is another major complication, ${ }^{9,19}$ which might be associated with deltoid dysfunction (such as deltoid paralysis and partially absence of deltoid) and relatively short prosthesis. To improve the shoulder stability, the axillary nerve was protected carefully and a custom-made humerus prosthesis was used to reconstruct the defects following the tumour resection. In the latest follow-up, no instability was observed in our patients. Recently, the reverse allograft-prosthetic composite has been used for stability improvement after resection of the proximal humerus tumours. ${ }^{9,10}$ However, the complications caused by the large allograft should be considered. ${ }^{9}$ Of note, we also noticed that Zou et $\mathrm{al}^{25}$ successfully used a 3D-printed shoulder prosthesis to reconstruct the patient's severe bone defects in the revision of total shoulder arthroplasty. Although the 3D-printed humerus prosthesis may facilitate the soft tissue attachment, a custom-made humerus prosthesis was selected in this study because of the higher price of 3D-printed prosthesis.

However, admittedly, the 3D printing technology still faces numerous challenges and possesses certain limitations, which restrict its widespread application. At present, the process of $3 \mathrm{D}$ printing manufacturing is timeconsuming and is not suitable for emergency cases. ${ }^{42}$ Fortunately, with incessant improvement of the 3D printing technology, the personalized 3D-printed prosthesis can now be designed and manufactured in 7-10 days in our team. However, the personalized 3D-printed prosthesis is expensive and not all patients can afford it. ${ }^{34}$ We believe that with the rapid development of the productivity and digital technology, the 3D printing technology will become affordable and be more widely available in the coming decade.

Several limitations of our study should be considered. First, the retrospective study with a relatively short followup period is insufficient to account for the long-term efficacy of this promising prosthesis. Second, the number of cases in this group was relatively small. Despite the small sample size, our study provides a new therapeutic alternative for reconstruction after en bloc resection of the proximal humerus tumours.

\section{Conclusion}

The 3D-printed guiding baseplate facilitated the accurate implantation of the glenoid prosthesis. The RSA based on a 3D-printed glenoid prosthesis and a personalized custom-made humerus prosthesis significantly improved the shoulder function and decreased the complication rate. Further studies of a larger scale as well as with longterm follow-up remain necessary to validate this therapeutic option.

\section{Acknowledgments}

This study was supported by Grants 2016YFC1100100 from The National Key Research and Development Program of China, Grants 91649204 from Major Research Plan of National Natural Science Foundation of China.

\section{Disclosure}

The authors declare that they have no conflict of interest.

\section{References}

1. Asavamongkolkul A, Waikakul S, Phimolsarnti R, Kiatisevi P, Wangsaturaka P. Endoprosthetic reconstruction for malignant bone and soft-tissue tumors. J Med Assoc Thai. 2007;90(4):706-717. 
2. Liu T, Zhang Q, Guo X, Zhang X, Li Z, Li X. Treatment and outcome of malignant bone tumors of the proximal humerus: biological versus endoprosthetic reconstruction. BMC Musculoskelet Disord. 2014;15:69. doi:10.1186/1471-2474-15-69

3. Wang B, Wu Q, Liu J, Yang S, Shao Z. Endoprosthetic reconstruction of the proximal humerus after tumour resection with polypropylene mesh. Int Orthop. 2015;39(3):501-506. doi:10.1007/s00264-014-2597-2

4. Bilgin SS. Reconstruction of proximal humeral defects with shoulder arthrodesis using free vascularized fibular graft. $J$ Bone Joint Surg Am. 2012;94(13):e94. doi:10.2106/JBJS.J.01823

5. DeGroot H, Donati D, Di Liddo M, Gozzi E, Mercuri M, The use of cement in osteoarticular allografts for proximal humeral bone tumors. Clin Orthop Relat Res. 2004;(427):190-197. doi:10.1097/01. blo.0000138959.50057.2c

6. Rodl RW, Gosheger G, Gebert C, Lindner N, Ozaki T, Winkelmann W. Reconstruction of the proximal humerus after wide resection of tumours. J Bone Joint Surg Br. 2002;84(7):1004-1008. doi:10.1302/0301-620X.84B7.0841004

7. Sharma S, Turcotte RE, Isler MH, Wong C. Experience with cemented large segment endoprostheses for tumors. Clin Orthop Relat Res. 2007;459:54-59. doi:10.1097/BLO.0b013e3180514c8e

8. Torbert JT, Fox EJ, Hosalkar HS, Ogilvie CM, Lackman RD. Endoprosthetic reconstructions: results of long-term followup of 139 patients. Clin Orthop Relat Res. 2005;438:51-59. doi:10.1097/ 01.blo.0000179735.37089.c2

9. King JJ, Nystrom LM, Reimer NB, Gibbs CP Jr., Scarborough MT, Wright TW. Allograft-prosthetic composite reverse total shoulder arthroplasty for reconstruction of proximal humerus tumor resections. J Shoulder Elb Surg. 2016;25(1):45-54. doi:10.1016/j.jse.2015.06.021

10. Lazerges C, Dagneaux L, Degeorge B, Tardy N, Coulet B, Chammas M. Composite reverse shoulder arthroplasty can provide good function and quality of life in cases of malignant tumour of the proximal humerus. Int Orthop. 2017;41(12):2619-2625. doi:10.1007/ s00264-017-3538-7

11. Sanchez-Sotelo J, Wagner ER, Sim FH, Houdek MT. Allograftprosthetic composite reconstruction for massive proximal humeral bone loss in reverse shoulder arthroplasty. J Bone Joint Surg Am. 2017;99(24):2069-2076. doi:10.2106/JBJS.16.01495

12. Maclean S, Malik SS, Evans S, Gregory J, Jeys L. Reverse shoulder endoprosthesis for pathologic lesions of the proximal humerus: a minimum 3-year follow-up. J Shoulder Elb Surg. 2017;26 (11):1990-1994. doi:10.1016/j.jse.2017.04.005

13. Sirveaux F. Reconstruction techniques after proximal humerus tumour resection. Orthop Traumatology Surg Res. 2019;105(1S): S153-S164. doi:10.1016/j.otsr.2018.04.024

14. Boileau P, Watkinson DJ, Hatzidakis AM, Balg F. Grammont reverse prosthesis: design, rationale, and biomechanics. J Shoulder Elb Surg. 2005;14(1Suppl S):147S-161S. doi:10.1016/j.jse.2004.10.006

15. Nolan BM, Ankerson E, Wiater JM. Reverse total shoulder arthroplasty improves function in cuff tear arthropathy. Clin Orthop Relat Res. 2011;469(9):2476-2482. doi:10.1007/s11999-010-1683-z

16. Boileau P, Watkinson D, Hatzidakis AM, Hovorka I. Neer award 2005: the Grammont reverse shoulder prosthesis: results in cuff tear arthritis, fracture sequelae, and revision arthroplasty. J Shoulder Elb Surg. 2006;15(5):527-540. doi:10.1016/j.jse.2006.01.003

17. Cuff DJ, Pupello DR. Comparison of hemiarthroplasty and reverse shoulder arthroplasty for the treatment of proximal humeral fractures in elderly patients. J Bone Joint Surg Am. 2013;95(22):2050-2055. doi:10.2106/JBJS.L.01637

18. Kaa AK, Jorgensen PH, Sojbjerg JO, Johannsen HV. Reverse shoulder replacement after resection of the proximal humerus for bone tumours. Bone Joint J. 2013;95-B(11):1551-1555. doi:10.1302/0301-620X.95B 11.31545

19. Bonnevialle N, Mansat P, Lebon J, Laffosse JM, Bonnevialle P. Reverse shoulder arthroplasty for malignant tumors of proximal humerus. J Shoulder Elb Surg. 2015;24(1):36-44. doi:10.1016/j.jse.2014.04.006
20. Fang C, Cai H, Kuong E, et al. Surgical applications of three-dimensional printing in the pelvis and acetabulum: from models and tools to implants. Unfallchirurg. 2019;122(4):278-285. doi:10.1007/s00113-019-0626-8

21. Wang B, Hao Y, Pu F, Jiang W, Shao Z. Computer-aided designed, three dimensional-printed hemipelvic prosthesis for peri-acetabular malignant bone tumour. Int Orthop. 2018;42(3):687-694. doi:10.1007/s00264-017-3645-5

22. Enneking WF, Dunham W, Gebhardt MC, Malawar M, Pritchard DJ. A system for the functional evaluation of reconstructive procedures after surgical treatment of tumors of the musculoskeletal system. Clin Orthop Relat Res. 1993;286:241-246.

23. Davis AM, Wright JG, Williams JI, Bombardier C, Griffin A, Bell RS. Development of a measure of physical function for patients with bone and soft tissue sarcoma. Qual Life Res. 1996;5(5):508-516. doi:10.1007/BF00540024

24. Glanzmann MC, Goldhahn J, Flury M, Schwyzer HK, Simmen BR. Deltoid flap reconstruction for massive rotator cuff tears: mid- and long-term functional and structural results. J Shoulder Elb Surg. 2010;19(3):439-445. doi:10.1016/j.jse.2009.06.005

25. Zou Y, Yang Y, Han Q, et al. Novel exploration of customized 3D printed shoulder prosthesis in revision of total shoulder arthroplasty: a case report. Medicine. 2018;97(47):e13282. doi:10.1097/MD.0000000000013282

26. Chalmers PN, Keener JD. Expanding roles for reverse shoulder arthroplasty. Curr Rev Musculoskelet Med. 2016;9(1):40-48. doi:10.1007/s12178-016-9316-0

27. Marulanda GA, Henderson E, Cheong D, Letson GD. Proximal and total humerus reconstruction with the use of an aortograft mesh. Clin Orthop Relat Res. 2010;468(11):2896-2903. doi:10.1007/s11999-010-1418-1

28. Gosheger G, Hillmann A, Lindner N, et al. Soft tissue reconstruction of megaprostheses using a trevira tube. Clin Orthop Relat Res. 2001; (393):264-271. doi:10.1097/00003086-200112000-00030.

29. Ji T, Tang X, Guo W. Enhancing soft-tissue reattachment in proximal humeral endoprosthetic reconstruction. J Orthop Surg. 2014;22 (1):100-103. doi:10.1177/230949901402200125

30. Flury MP, Frey P, Goldhahn J, Schwyzer HK, Simmen BR. Reverse shoulder arthroplasty as a salvage procedure for failed conventional shoulder replacement due to cuff failure-midterm results. Int Orthop. 2011;35(1):53-60. doi:10.1007/s00264-010-0990-z

31. Ackland DC, Patel M, Knox D. Prosthesis design and placement in reverse total shoulder arthroplasty. J Orthop Surg Res. 2015;10:101. doi:10.1186/s13018-015-0244-2

32. Diment LE, Thompson MS, Bergmann JHM. Clinical efficacy and effectiveness of 3D printing: a systematic review. BMJ Open. 2017;7 (12):e016891. doi:10.1136/bmjopen-2017-016891

33. Zadpoor AA, Malda J. Additive manufacturing of biomaterials, tissues, and organs. Ann Biomed Eng. 2017;45(1):1-11. doi:10.1007/ s10439-016-1719-y

34. Riggs KW, Dsouza G, Broderick JT, Moore RA, Morales DLS. 3Dprinted models optimize preoperative planning for pediatric cardiac tumor debulking. Transl Pediatr. 2018;7(3):196-202. doi:10.21037/ tp.2018.06.01

35. Wang F, Zhu J, Peng X, Su J. The application of 3D printed surgical guides in resection and reconstruction of malignant bone tumor. Oncol Lett. 2017;14(4):4581-4584. doi:10.3892/ol.2017.6749

36. Li X, Wang Y, Zhao Y, Liu J, Xiao S, Mao K. Multilevel 3D printing implant for reconstructing cervical spine with metastatic papillary thyroid carcinoma. Spine. 2017;42(22):E1326-E1330. doi:10.1097/ BRS.0000000000002229

37. Lu M, Li Y, Luo Y, Zhang W, Zhou Y, Tu C. Uncemented three-dimensional-printed prosthetic reconstruction for massive bone defects of the proximal tibia. World J Surg Oncol. 2018;16 (1):47. doi:10.1186/s12957-018-1333-6

38. Lu M, Min L, Xiao C, et al. Uncemented three-dimensional-printed prosthetic replacement for giant cell tumor of distal radius: a new design of prosthesis and surgical techniques. Cancer Manag Res. 2018;10:265-277. doi:10.2147/CMAR.S146434 
39. Zumstein MA, Pinedo M, Old J, Boileau P. Problems, complications, reoperations, and revisions in reverse total shoulder arthroplasty: a systematic review. J Shoulder Elb Surg. 2011;20(1):146-157. doi:10.1016/j.jse.2010.08.001

40. Levigne C, Boileau P, Favard L, et al. Scapular notching in reverse shoulder arthroplasty. J Shoulder Elb Surg. 2008;17(6):925-935. doi:10.1016/j.jse.2008.02.010

41. Friedman RJ, Barcel DA, Eichinger JK. Scapular notching in reverse total shoulder arthroplasty. J Am Acad Orthop Surg. 2019;27 (6):200-209. doi:10.5435/JAAOS-D-17-00026
42. Byrne N, Velasco Forte M, Tandon A, Valverde I, Hussain T. A systematic review of image segmentation methodology, used in the additive manufacture of patient-specific 3D printed models of the cardiovascular system. JRSM Cardiovasc Dis. 2016;5:2048004016645467. doi:10.1177/ 2048004016645467

\section{Publish your work in this journal}

Cancer Management and Research is an international, peer-reviewed open access journal focusing on cancer research and the optimal use of preventative and integrated treatment interventions to achieve improved outcomes, enhanced survival and quality of life for the cancer patient.
The manuscript management system is completely online and includes a very quick and fair peer-review system, which is all easy to use. Visit http://www.dovepress.com/testimonials.php to read real quotes from published authors. 